\title{
Numerical Analysis of Heat Storage Phenomenon In a Dual Latent Heat Sink
}

\author{
Krishna M. Kota ${ }^{1}$, Louis C. Chow ${ }^{2}$, Jianhua Du ${ }^{3}$, Jayanta S. Kapat ${ }^{4}$ \\ Department of Mechanical, Materials and Aerospace Engineering, \\ University of Central Florida, Orlando, FL 32816-2450, USA \\ Quinn H. Leland ${ }^{5}$ \\ Air Force Research Laboratory, Wright Patterson Air Force Base, Dayton, OH 45433, USA \\ and \\ Richard J. Harris ${ }^{6}$ \\ University of Dayton Research Institute, Dayton, OH 45469, USA
}

\begin{abstract}
This paper presents the concept of a dual latent heat sink intended for low thermal duty cycle electronic heat sink applications. The effectiveness of this heat sink depends on the rapidness of the heat storage facility in the design during the pulse heat generation period of the duty cycle. Heat storage in this heat sink involves transient simultaneous laminar film condensation of vapor and melting of an encapsulated phase change material in graphite foam. The key focus of this paper is to numerically analyze this conjugate heat transfer problem including the wall inertia effect and thus verify the effectiveness of the heat storage mechanism of the heat sink. An effective heat capacity formulation is employed for modeling the phase change problem and is solved using finite element method. The results of the developed model showed that the concept is effective in preventing undue temperature rise of the heat source.
\end{abstract}

\section{Nomenclature}

$=$ surface area of foam $\left(\mathrm{m}^{2}\right)$

$=$ specific surface area of the porous medium $\left(\mathrm{m}^{2} / \mathrm{m}^{3}\right)$

$=$ Biot number

$=$ effective specific heat of PCM and foam composite $(\mathrm{J} / \mathrm{kg} \cdot \mathrm{K})$

$=$ specific heat of foam $(\mathrm{J} / \mathrm{kg} \cdot \mathrm{K})$

$=$ specific heat of liquid phase of PCM $(\mathrm{J} / \mathrm{kg} \cdot \mathrm{K})$

$=$ latent heat of fusion of PCM averaged over the melting range $(\mathrm{J} / \mathrm{kg} \cdot \mathrm{K})$

$=$ specific heat of solid phase of PCM $(\mathrm{J} / \mathrm{kg} \cdot \mathrm{K})$

$=$ specific heat of TES unit column wall $(\mathrm{J} / \mathrm{kg} . \mathrm{K})$

$=$ pore diameter (micrometers)

$=$ convection heat transfer coefficient $\left(\mathrm{W} / \mathrm{m}^{2} . \mathrm{K}\right)$

$=$ foam ligament and PCM interfacial heat transfer coefficient $\left(\mathrm{W} / \mathrm{m}^{2} . \mathrm{K}\right)$

$=$ latent heat of vaporization of the condensate $(\mathrm{J} / \mathrm{kg})$

$=$ latent heat of fusion of the $\mathrm{PCM}(\mathrm{J} / \mathrm{kg})$

$=$ latent heat of fusion of the PCM in the operating temperature range $(\mathrm{J} / \mathrm{kg})$

$=$ Jakob number

\footnotetext{
${ }^{1}$ Student, AIAA Member

${ }^{2}$ Professor and University Chair, AIAA Associate Fellow

${ }^{3}$ Research Professor

${ }^{4}$ Lockheed Martin Professor, AIAA Associate Fellow

${ }_{6}^{5}$ Senior Mechanical Engineer, AIAA Member

${ }^{6}$ Research Engineer, AIAA Member
}

American Institute of Aeronautics and Astronautics 


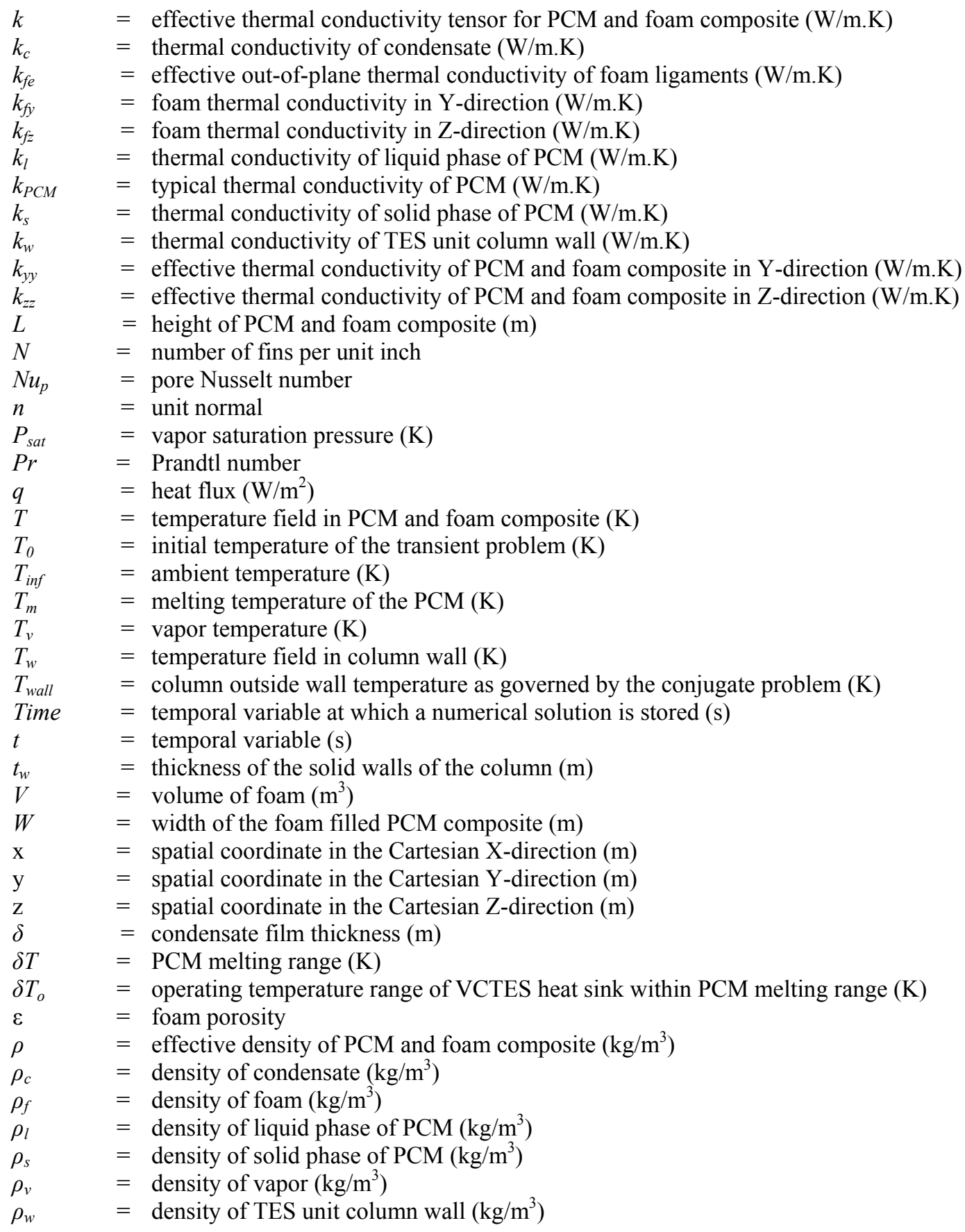

\section{Introduction}

Future electronic systems will involve small size, light-weight and compact components that release very high waste heat in a pulse. Miniaturization of such systems will generate large heat fluxes during the pulse time, which will require the development of an efficient thermal management system.

This paper introduces the concept of a unique dual latent heat sink intended for low thermal duty cycle electronic heat sink applications. This new heat sink design combines the features of a vapor chamber with rapid thermal energy storage (TES) employing graphite foam inside the heat storage facility along with phase change materials. As against to using a heat pipe for heat acquisition and transport, a vapor chamber acting as a heat spreader enables for more uniform temperature distribution along the surface of the device being cooled, still incorporating the rapid heat absorption feature of a heat pipe. Hence, the vapor chamber feature elevates the system thermal conductivity by 
about hundred to thousand times that of pure metallic copper. This feature coupled with the rapid TES facility in the design makes this integrated system an useful one for multiple thermal management applications. Potential application areas include but are not limited to temperature control of IGBTs and MOSFETs, electronic cooling in aircraft and avionics, intermittent high-speed machine element cooling, high-speed laser component cooling, high power microwave and missile launch applications and thermal management of electrical and electronic systems in hybrid electric vehicles.

\section{Concept of Integrated Vapor Chamber - Thermal Energy Storage (VCTES) System}

Complete details of the concept of the VCTES heat sink can be found in Ref. 1. It is a 7 MJ heat sink (Fig. 1) with a volume of $0.072 \mathrm{~m}^{3}$ and weighing $57.5 \mathrm{~kg}$. It is designed to have a low vapor-to-condenser temperature difference $\left(7{ }^{\circ} \mathrm{C}\right)$, fast charging ability, environment safe operation, high heat flux capability $\left(500 \mathrm{~W} / \mathrm{cm}^{2}\right.$ over an area of $\left.100 \mathrm{~cm}^{2}\right)$ and high energy storage density $\left(97 \mathrm{MJ} / \mathrm{m}^{3}, 0.122 \mathrm{MJ} / \mathrm{kg}\right.$ ) over a charging pulse of $140 \mathrm{~s}$. A scaleddown version of this dual latent heat sink is being tested at the University of Central Florida and this paper focuses on numerically simulating the heat storage phenomenon as applied to this scaled-down version.

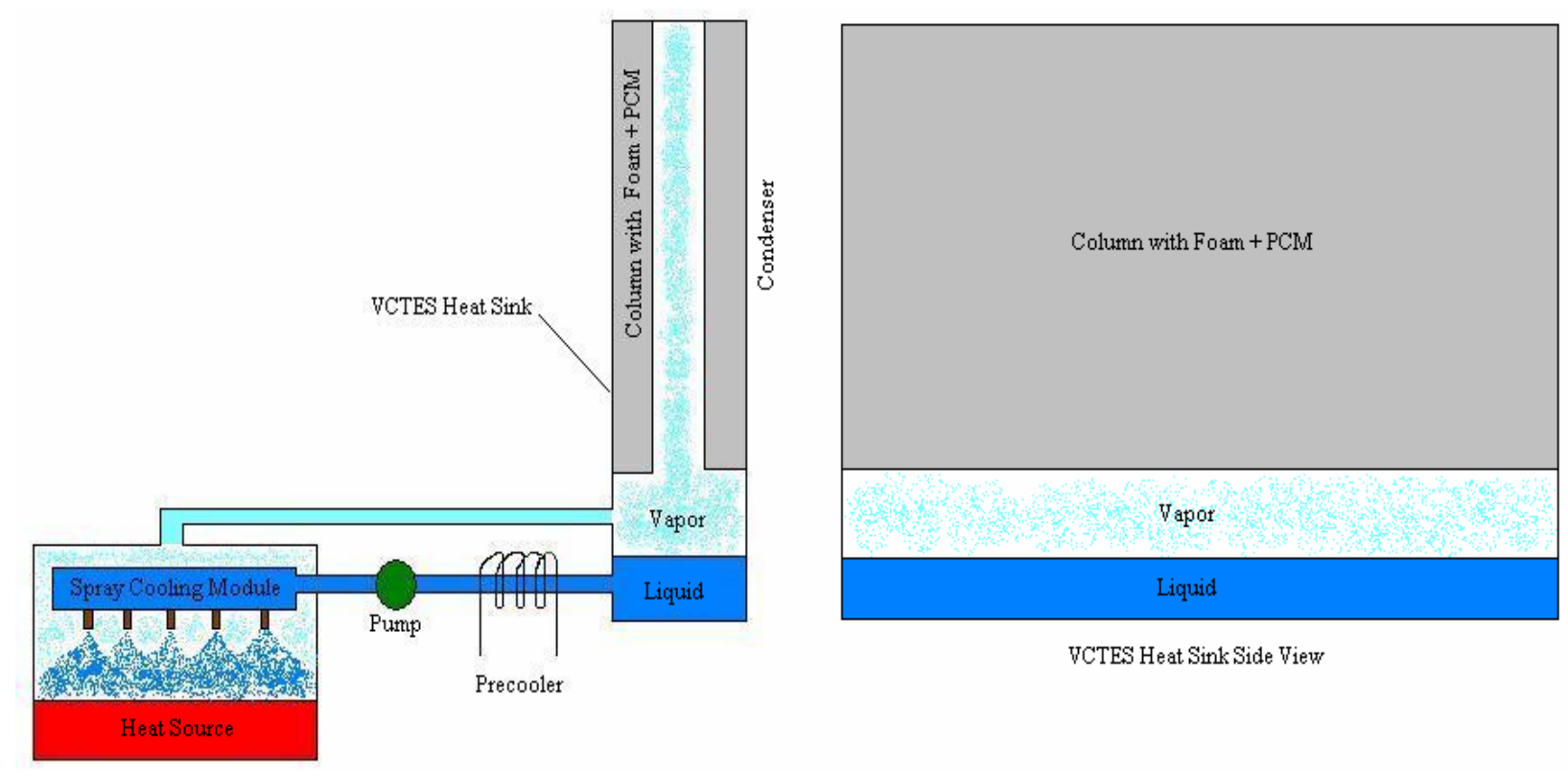

Figure 1. Concept of integrated VCTES system (applied to a spray-cooling module)

This dual latent heat sink system thermal operation is characterized by the following three processes namely,

a) Heat Acquisition (by liquid-vapor phase change inside the vapor chamber)

b) Heat Storage (by vapor flow followed by film condensation on containers with simultaneous phase change of a PCM inside those containers) and,

c) Heat Rejection (by discharging through the condenser surface of the vapor chamber).

Of these, the most important process is the heat storage or more precisely, the swift temporary storage of waste heat using encapsulated phase change materials in a conductive medium. It is because of this rapid temporary thermal energy storage that this heat sink can be designed for an average heat load rather than for pulsed heat loads. But as most of the available phase change materials have very low thermal conductivities, the heat storage path evolves as the maximum heat resistance path in heat sinks employing thermal energy storage. But the key feature that enables for a fast heat storage mechanism in this heat sink is a coupled or conjugate heat transfer phenomenon wherein the vapor carries heat from the source and condenses on the outside of thermal energy storage containers and the heat is simultaneously absorbed by phase change materials encapsulated inside those containers in a highly thermally conductive porous graphite foam. Hence, the key focus of this paper is to numerically analyze the key phenomenon of heat storage as specific to the above-mentioned heat sink.

Most thermal energy storage modeling till date focused only on the heat absorption process with a constant temperature or constant heat flux condition imposed on the surrounding boundary/encapsulation. This kind of 
analysis can produce different conclusions compared to actual situation in the above-mentioned integrated VCTES heat sink, where the wall boundary condition for the PCM melting comes from film condensation of vapor on the outer surface of container walls.

There is no available literature till date that focused on the conjugate heat transfer problem of transient laminar film condensation coupled with encapsulated PCM melting in porous graphite foams. To the best knowledge of the authors, only limited attention was focused on the numerical analysis of conjugate transient heat transfer problem of condensation and melting. Contreras and Thorsen ${ }^{2}$ were probably the first to analytically study the transient condensation of a saturated vapor on a solid of same chemical composition coupled with melting of the same solid. They ignored the inertia terms in the momentum equation but included the convective terms in the energy equation and obtained an integral solution using a quadratic temperature profile. Galamba and Dhir ${ }^{3-5}$ numerically analyzed the problem of transient condensation of saturated vapor on both normal ${ }^{3}$ and subcooled ${ }^{4}$ vertical solid walls whose melting temperatures were less than the vapor saturation temperature. The condensing fluid and the melting wall material were assumed immiscible. They used both analytical and numerical techniques for solving the governing equations, which ignored inertia terms in the momentum equation and convective terms in the energy equation. The melt layer physics is treated in a similar way as the condensate film using analogous parameters for melt layer thickness and steady-state time as for film condensation. This kind of treatment for melting does not hold good if melting occurs in porous media like foams, especially in most metal and graphite foams where the pore size is in the order of a few tens to hundreds of microns. The small pore size in foams will provide a capillary effect and lower the effect of gravity. In addition, it was shown in Ref. 6 that there will be practically a minimal temperature difference between the ligaments of a foam and PCM in the pores if the surface area to volume ratio $A / V$ for the foam is more than $1575 \mathrm{~m}^{2} / \mathrm{m}^{3}$. For graphite foams, $A / V$ is typically of the order of $20,000\left(\mathrm{~m}^{2} / \mathrm{m}^{3}\right)^{7}$. These conditions specific to graphite foams will provide a Rayleigh number smaller than the critical value for natural convection in the melt to become significant. Hence, the PCM melting in graphite foams must be modeled as transient conduction dominated moving melt front problem. Further, the encapsulation of PCM in containers induces wall effects that were not included in any of the prior related work. Chen and Chang ${ }^{8}$ studied an analogous conjugate problem of laminar film condensation and natural convection on opposite sides of a vertical wall and included the effect of wall thermal resistance. Char and $\operatorname{Lin}^{9}$ extended the problem by considering the two media separated by the wall as porous. Both Refs. 8 and 9 were steady state analyses.

Hence, it forms an interesting aspect to numerically solve and understand the transient problem of laminar film condensation coupled with encapsulated solid-liquid PCM phase change inside graphite foam. This would help in better understanding and quantifying important parameters that govern the rapidity and efficiency of such temporary heat storage mechanisms in future as in the current VCTES system.

\section{Numerical Model}

As mentioned before, a fast design tool for this VCTES system was already developed to facilitate a preliminary design for the experiment and this numerical simulation is based on the geometry as a result of optimizing the design tool. Full details of optimization and theoretical aspects of the design tool can be found in Ref. 1. Individual numerical models for PCM melting inside graphite foam and film condensation on the outside of PCM encapsulating containers are given below. Coupling of the two models with heat conduction within the TES container walls is also mentioned.

\section{A. Encapsulated PCM phase change in a high thermally conductive porous graphite foam}

The phase change process of PCM in highly thermally conductive porous graphite foam is solved as a nonlinear transient moving boundary problem. It was observed that the two-temperature model ${ }^{10}$ as observed in metal foams can be solved as a one-temperature model ${ }^{6}$ in graphite foams owing to the large $A / V$ ratio in graphite foams. The reason for this is explained below.

The actual mode of heat transfer to the PCM in a pore can be assumed as three dimensional through conduction in the foam ligaments. This assumption is equivalent to opening up a cuboid (a hollow rectangular fin) lying in a three dimensional Cartesian coordinate system into a surface (fin) lying in a two-dimensional coordinate system and assuming that the PCM lies above this fin with a uniform thickness. But when the surface is closed back to form a cuboid, there will be overlapping of PCM volumes. So a two-dimensional model provides for lesser foam surface area for PCM phase change compared to a cuboid fin model. But this increase in foam surface area in the cuboid fin model can be alternately captured in the two-dimensional fin model by increasing the number of fins per unit inch . On increasing $N$ above 20, it was shown in Ref. 6 that the PCM phase change in porous foams can be modeled using a one-temperature model. This is also true because of a very low heat flux at the pore level when $N$ is a large 
number. So, it is accurate enough to use a two-dimensional one-temperature model to model phase change in foams with high $N$. While $N=20$ corresponds to a surface area to volume ratio of $1575\left(\mathrm{~m}^{2} / \mathrm{m}^{3}\right)$ in Ref. 6 , Pocofoam ${ }^{\circledR}$ with a typical surface area to volume ratio of $20,000\left(\mathrm{~m}^{2} / \mathrm{m}^{3}\right)^{7}$ leads to a value of $N$ much greater than 20 . Therefore, a single temperature representative of both the foam and PCM temperatures can be used in the numerical modeling.

Another interesting feature to be noted with respect to heat transfer in graphite foams filled with a PCM is that the heat transfer occurs in series from the column (TES unit) wall to the column center rather than in a lumped manner in all the foam ligaments first and then in parallel paths in all the pores. This can be better explained by considering an equivalent Biot number (Bi) as given by Eq. (14) of Lee and Vafai ${ }^{11}$.

$$
B i=\frac{h_{\text {int }} \gamma a W^{2}}{4 k_{f e}}
$$

$\gamma=1$ for parallel plate $/$ rectangular configurations ${ }^{11}$.

For Pocofoam ${ }^{\circledR}$ enclosed in a container as shown in Fig. $2, a=20,000^{7}, W / 2=0.0064^{1}$, and $\mathrm{k}_{\mathrm{fe}}=135^{12}$ units. $h_{\text {int }}$ can be found from the pore Nusselt number as,

$$
N u_{p}=\left(h_{\text {int }} \cdot d_{p} / k_{P C M}\right)
$$

where $d_{p}=350$ micrometers for Pocofoam ${ }^{12}$ and $k_{P C M}$ can be assumed as $0.2 \mathrm{~W} / \mathrm{m} . \mathrm{K}$ (representative of most paraffin waxes). $N u_{p}$ can be assumed as $2^{13}$ by approximating the shape of the PCM interacting with the solid foam as spheres surrounded by Pocofoam ${ }^{\circledR}$. This gives, $h_{\text {int }}=1143 \mathrm{~W} / \mathrm{m}^{2} . \mathrm{K}$.

Using the above values in Eq. (1), we get $\mathrm{Bi} \sim 7$. With a $\mathrm{Bi}>>0.1$, it is clear that the conduction resistance in the foam ligaments is much higher compared to the convective heat transfer resistance at the interface of the foam ligaments and PCM, which implies the temperature gradient inside foam ligaments cannot be ignored. Hence, the heat transfer occurs in series from the container wall to the container center portion rather than in the foam ligaments first followed by parallel paths in all the pores.

As far as the effect of film condensation on the dimensions of the phase change problem is concerned, it must be noted that the column wall temperature will have a variation along the height and the temperature drop across the film in the cross-stream direction will be about the same or even more compared to the temperature drop in the PCM filling the foam. Therefore, the heat transfer in PCM will be two-dimensional.

Based on the above conclusions, the model then collapses to solving a phase change problem in the Y-Z plane of the TES column (Fig. 2) by considering the effective thermophysical properties of the foam-PCM composite based on the foam porosity. A fixed grid heat capacity formulation ${ }^{14-16}$ is used to model this problem. Enthalpy models $\mathrm{s}^{17-20}$ for phase change are attractive in the sense they do not require explicit tracking of the phase change interface unlike in the heat capacity methods. But, whereas the heat capacity method provides explicit temperature field in the phase change domain, the temperature field in an enthalpy model has to be implicitly evaluated using the enthalpytemperature correlation. In addition, liquid fraction field is imbibed in the temperature field in the enthalpy models. It has been traditionally observed that for phase change problems with a melting range and for conjugate heat transfer problems involving adjacent walls, heat capacity method performs better ${ }^{16}$. Therefore, for the current simulation, an easy-to-implement modified effective heat capacity method is used.

The mathematical model is described below and includes the following assumptions:

a) The effects of natural convection within the molten PCM can be ignored as explained earlier. Therefore, the phase change process is conduction dominated.

b) Temperature gradients in the X-direction (Fig. 2, which is representative of actual columns in the scaleddown that is being tested at University of Central Florida) can be ignored owing to symmetry of the boundary conditions with respect to that direction. This can be justified since the X-direction boundary conditions for the phase change inside the column come from the condensing film on the outside of the column and the condensate thickness does not vary along the X-direction at any time during the TES unit charging process.

c) Thermal conductivity of PCM is different for solid and liquid phases but independent of temperature in any one phase. This assumption is reasonable especially for heavy paraffin waxes (number of carbon atoms $>$ 30 ) that will be used in VCTES experiments of the current work. Paradela et al ${ }^{21}$ exclusively modeled the thermal conductivities of paraffins that are primarily used for TES. From Eq. (9) and Table I in Ref. 21, it can be seen that the first and second order temperature coefficients for most paraffins are very small compared to their zero order coefficient. In addition, it can be observed that as the value of number of carbon atoms increases, the first and second order temperature coefficients decrease. 
d) Density of PCM is different for solid and liquid phases but independent of temperature in any one phase.

e) PCM is homogeneous and isotropic and has no property degradation with time.

For conduction controlled PCM phase change, the transient energy equation in Cartesian coordinate system in general is given by,

$$
c_{p}(T) \frac{\partial T(x, y, z, t)}{\partial t}=\nabla \cdot\left(\frac{k}{\rho} \nabla T(x, y, z, t)\right)
$$

Since graphite foams exhibit anisotropic thermal conductivity ${ }^{1}$, the numerical model includes the same by defining a two-dimensional tensor for $k$ as follows:

$$
[k]=\left[\begin{array}{cc}
k_{y y} & 0 \\
0 & k_{z z}
\end{array}\right]
$$

As applied to a column as shown in Fig. 2, the energy equation then becomes,

$$
c_{p}(T) \frac{\partial T(y, z, t)}{\partial t}=\left(k_{y y} \frac{\partial^{2} T(y, z, t)}{\partial y^{2}}+k_{z z} \frac{\partial^{2} T(y, z, t)}{\partial z^{2}}\right) \frac{1}{\rho}
$$

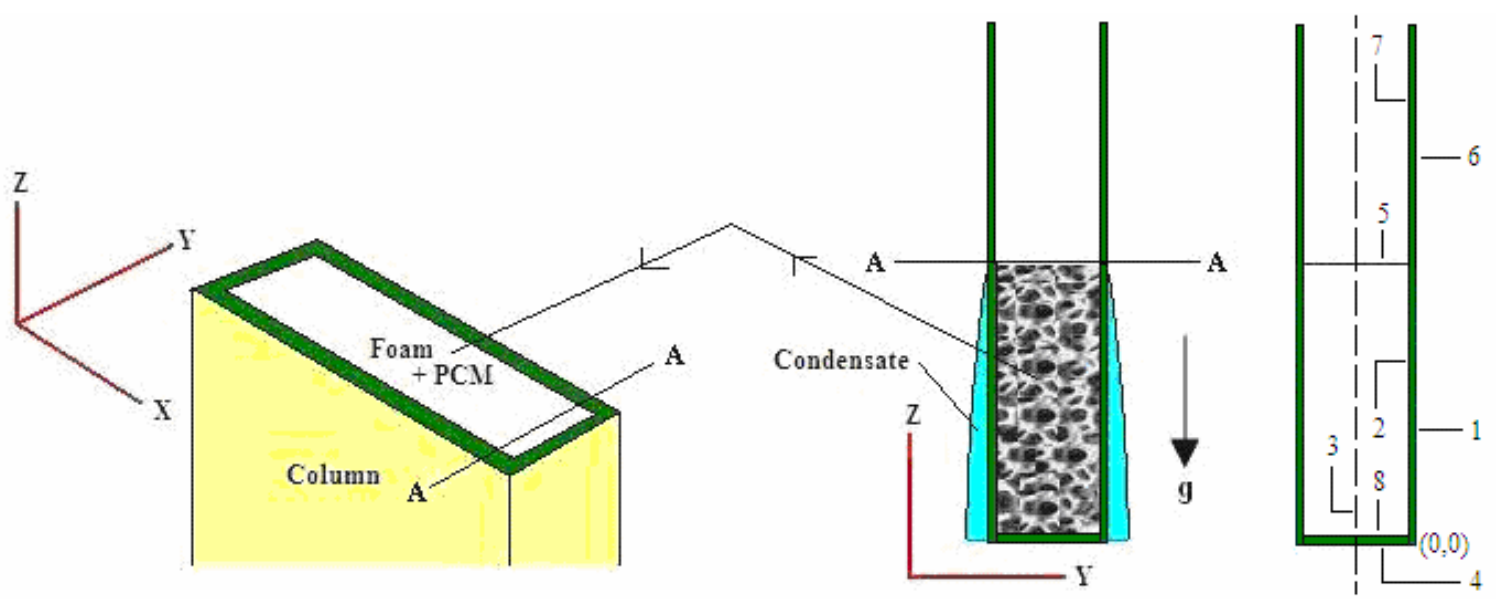

Figure 2. Schematic of the TES unit considered for numerical simulation

Since melting range is a usual characteristic of paraffin waxes and since they are used in the VCTES experiments of the current work, the specific heat is defined as shown in Eq. (6).

$$
c_{p}(T)=\left\{\begin{array}{c}
\varepsilon c_{p s}+(1-\varepsilon) c_{p f} ; T<T_{m}-(\delta T / 2) \\
\varepsilon\left(c_{p m}\right)+(1-\varepsilon) c_{p f} ; T_{m}-(\delta T / 2) \leq T \leq T_{m}+(\delta T / 2) \\
\varepsilon c_{p l}+(1-\varepsilon) c_{p f} ; T>T_{m}+(\delta T / 2)
\end{array}\right.
$$

During melting of PCM, the specific heat is modeled as given in Ref. 22.

$$
c_{p m}=\frac{h_{s f}}{\delta T}
$$

$\rho$ in Eq. (5) is given by Eq. (8).

\footnotetext{
${ }^{1}$ http://www.poco.com/MaterialsandServices/ThermalMaterials/POCOFoam/PropertiesCharacteristics/tabid/130/Default.aspx 


$$
\begin{gathered}
\rho=\left\{\begin{array}{c}
\varepsilon \rho_{s}+(1-\varepsilon) \rho_{f} ; T<T_{m}-(\delta T / 2) \\
\varepsilon\left(\frac{\rho_{s}+\rho_{l}}{2}\right)+(1-\varepsilon) \rho_{f} ; T_{m}-(\delta T / 2) \leq T \leq T_{m}+(\delta T / 2) \\
\varepsilon \rho_{l}+(1-\varepsilon) \rho_{f} ; T>T_{m}+(\delta T / 2)
\end{array}\right. \\
k_{y y}=\left\{\begin{array}{c}
\varepsilon k_{s}+(1-\varepsilon) k_{f y} ; T<T_{m}-(\delta T / 2) \\
\varepsilon\left(\frac{k_{s}+k_{l}}{2}\right)+(1-\varepsilon) k_{f y} ; T_{m}-(\delta T / 2) \leq T \leq T_{m}+(\delta T / 2) \\
\varepsilon k_{l}+(1-\varepsilon) k_{f y} ; T>T_{m}+(\delta T / 2)
\end{array}\right.
\end{gathered}
$$

$k_{z z}$ is defined similarly as in Eq. (9).

Initial Condition:

$$
T(y, z, 0)=T_{0}
$$

Boundary Conditions:
a) $q \cdot n=0$ on boundary 3
b) Temperature and heat flux continuity on boundaries 2 and 8
c) $q . n=h\left[T\left(y, L+t_{w}, t\right)-T_{i n f}\right]$ on boundary 5

\section{B. Laminar film condensation on a flat vertical surface}

Analysis of laminar transient film condensation on a vertical plate was done by Sparrow and Siegel ${ }^{23}$, Reed et al. $^{24}$ and Chung ${ }^{25}$. It was shown in Ref. 23 and Ref. 24 that the transient problem can be treated as quasi-steady provided that $J a$ and $J a / P r$ are both $<<1$. This implies that the temperature profile is linear and the velocity profile is parabolic.

It must be noted that in Ref. 24, it was shown that the above condition on $J a$ and $J a / P r$ holds good for water in atmospheric conditions, while the actual conditions in VCTES are designed to be not atmospheric ${ }^{1}$. In addition, Ja depends on the temperature difference across the liquid film, which differs from case to case. Hence, appropriate values for thermophysical properties of water for conditions in the vapor chamber ( $P_{\text {sat }}$ of approximately $\left.2 \mathrm{~atm}\right)$ were used to evaluate $J a$ and $\operatorname{Pr}$ for application to the current problem under consideration. Since the film thickness will be small, the temperature difference across the film will be small $(\sim 1 \mathrm{~K})$. It was found that $J a=0.0019$ and $J a / P r=0.0014$. Even for a temperature difference across the film of $10 \mathrm{~K}$ (which is highly unlikely), $J a$ and $\operatorname{Pr}$ are 0.019 and 0.014 , respectively with both much smaller than unity. Therefore, a quasi-steady approach can be used.

The film thickness in any given time-step can be reasonably approximated using Eq. (11) as shown below, where the variation in the wall temperature in the column height direction is also included.

$$
\delta(z, t)=\left[\frac{4 k_{c} \mu_{c} \int_{0}^{L}\left(T_{v}-T_{\text {wall }}(z, t)\right) d z}{h_{l v} g\left(\rho_{c}-\rho_{v}\right) \rho_{c}}\right]^{0.25}
$$

\section{Heat conduction in the solid wall encapsulating the PCM}

A two-dimensional energy equation in Cartesian coordinates was solved in the solid wall as shown in Eq. (12).

$$
c_{p w} \frac{\partial T_{w}(y, z, t)}{\partial t}=\left(\frac{\partial^{2} T_{w}(y, z, t)}{\partial y^{2}}+\frac{\partial^{2} T_{w}(y, z, t)}{\partial z^{2}}\right) \frac{k_{w}}{\rho_{w}}
$$

Initial Condition:

Boundary Conditions:

$$
T_{w}(y, z, 0)=T_{0}
$$


d) $q \cdot n=0$ on boundary 4

e) $T(0, z, t)=T_{\text {wall }}(z, t)$ on boundary 1

\section{Numerical coupling}

In the numerical problem, coupling is required at two junctions; between condensing film and the column outer wall (boundary 1 in Fig. 2) and between the column inner wall (boundary 2 in Fig. 2) and the composite region of graphite foam and PCM. Coupling is done using the temperature and heat flux continuity at both the interfaces.

The assumptions made for the numerical coupling are:

1) The vapor temperature ' $T_{v}$ ' is a constant.

2) The heat transfer coefficients for heat removal on the column boundaries 6 and 7 (Fig. 2) were chosen to be $50 \mathrm{~W} / \mathrm{m}^{2} \mathrm{~K}$ and $5 \mathrm{~W} / \mathrm{m}^{2} \mathrm{~K}$ for column outside and inside faces respectively.

The following solution procedure is implemented in each time-step:

1) Solve the PCM and graphite foam composite phase change problem.

2) Solve for $\delta(z, t)$.

3) Get $T_{\text {wall }}(z, t)$ using temperature and heat flux continuity at the column wall and condensate interface.

4) Go to the next time-step.

5) Repeat steps 1 through 4 until the end of the VCTES charging period.

Finite element method is used to solve the above system of equations. A commercial finite element code, COMSOL $\AA^{26}$ along with MATLAB ${ }^{27}$ is used and user-defined modules for solving PCM phase change in graphite foam and condensate film thickness are incorporated.

\section{Results and Discussion}

Since no experiments have been performed to check the validity of the current PCM phase change model, it is first verified with one of the experimental results as described in Ref. 28, wherein, a cylindrical copper block was attached to a cylindrical piece of POCO graphite foam. The copper block was made to act as a heat source by using an external heater attached to its top surface. The entire assembly was then immersed into a Teflon enclosure serving as thermal insulation. The graphite foam was a priori filled with paraffin wax. Schematic of the setup is shown in Fig. 3. Experiments were performed for different heat inputs and the transient temperature distribution of the PCM at the locations 2, 3, 4 of the foam and PCM composite are plotted in Fig. 8 of Ref. 28. A case where the heat input was $11.63 \mathrm{~W}$ is selected to validate the current model. The same setup and boundary conditions are implemented and the thermocouple locations 2, 3, 4 are picked to be exactly at the same locations as described in Ref. 28 and bear the same numbering. For a better comparison, the results are overlapped onto Fig. 8 in Ref. 28 and are shown in Fig. 4. It can be observed that the current model and assumptions can reasonably simulate PCM phase change in graphite foams. Since in Ref. 28, individual details of thermophysical properties of PCM, foam and heat transfer coefficient for natural convection were not mentioned, typical values for those as mentioned in literature were taken $\left(5 \mathrm{~W} / \mathrm{m}^{2} \mathrm{~K}\right.$ for the heat transfer coefficient ${ }^{13}$ ).
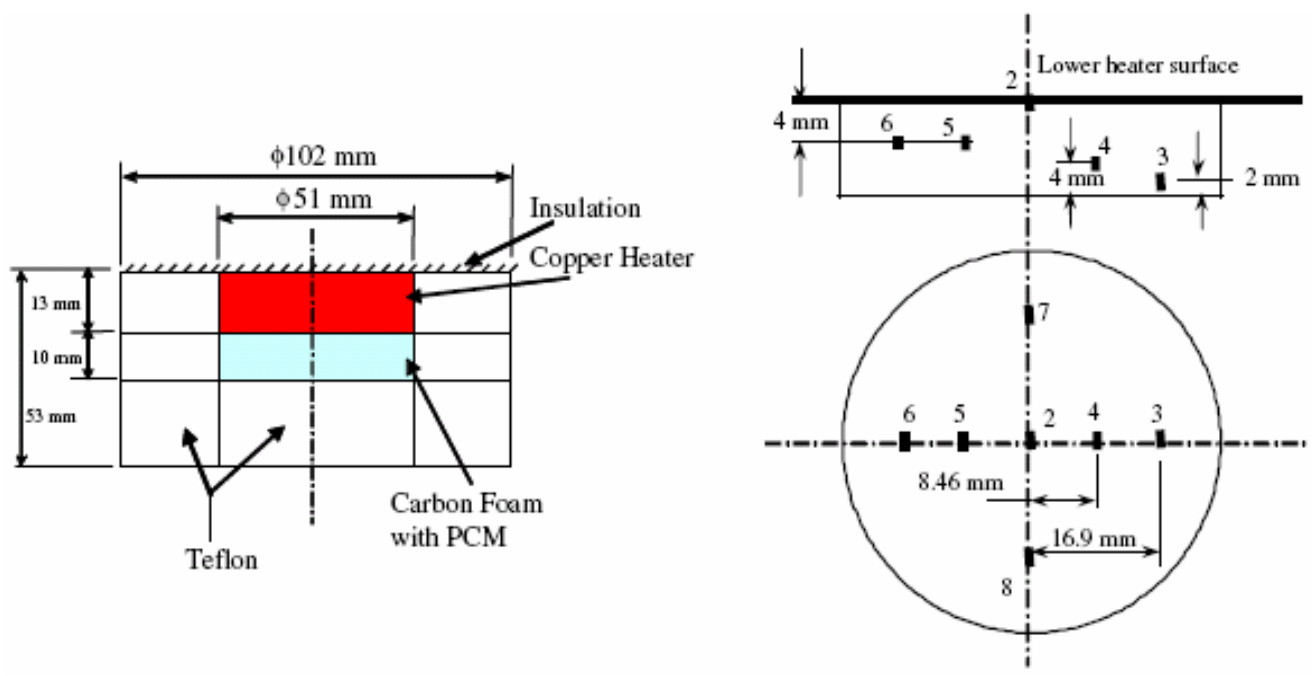

Figure 3. Schematic of thermal protection cell as given in Ref. 28 with thermocouple locations 


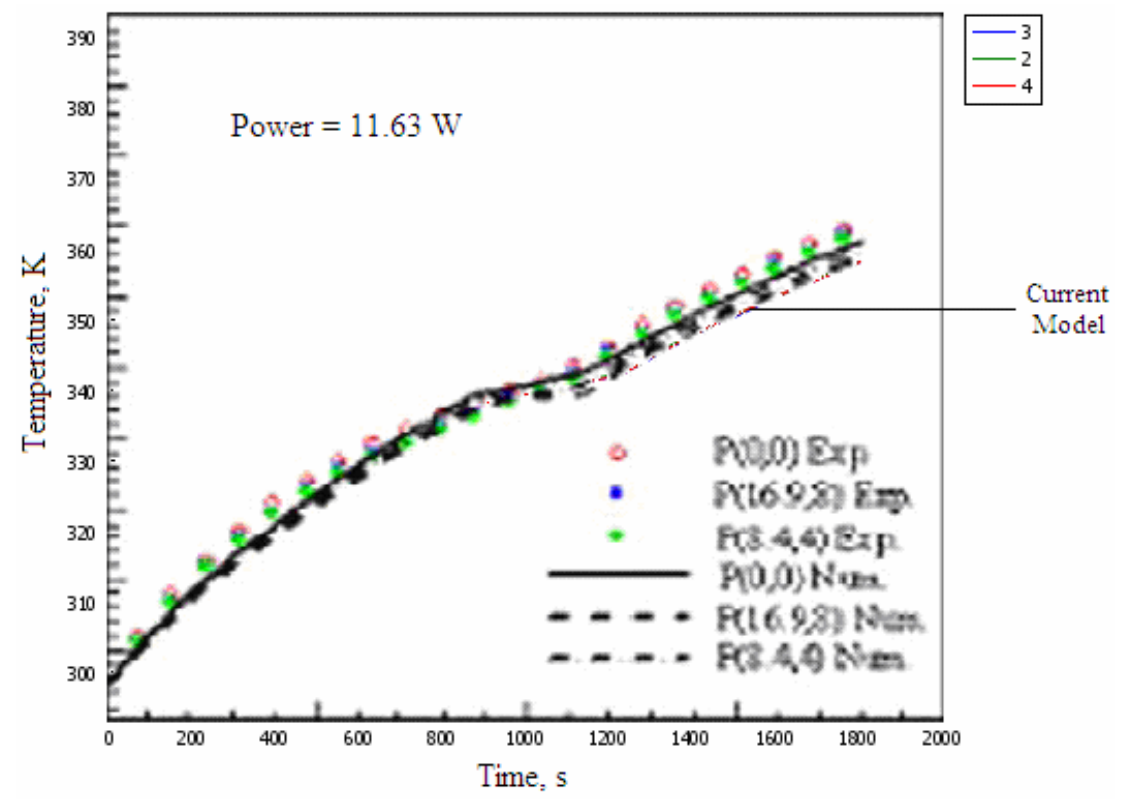

Figure 4. Comparison of current model with experimental results of Ref. 28

As mentioned before, the TES columns are designed for experiment and hence for future comparison purposes, the same dimensions ( $L=0.9$ ", $W=0.375$ " and $\left.t_{w}=0.064 "\right)$, material (copper) and thermocouple nomenclature are used for current numerical simulation.

The PCM is assumed as POLYWAX® 1000 and the DSC curve for the PCM is shown in Fig. 5. The following thermophysical properties are used in the numerical simulation: $c_{p s}=2900 \mathrm{~J} / \mathrm{kg} . \mathrm{K}, c_{p l}=3500 \mathrm{~J} / \mathrm{kg} . \mathrm{K}, h_{s f}=266 \mathrm{~kJ} / \mathrm{kg}$, $\rho_{s}=970 \mathrm{~kg} / \mathrm{m} 3$ and $\rho_{l}=900 \mathrm{~kg} / \mathrm{m} 3$. Since, thermal conductivities of solid and liquid phases of the wax are not known, typical values of thermal conductivities $\left(k_{s}=0.20 \mathrm{~W} / \mathrm{m} . \mathrm{K}, k_{l}=0.18 \mathrm{~W} / \mathrm{m} . \mathrm{K}\right)$ available in literature for most paraffin waxes are used. Corresponding values for graphite foam are taken as $c_{p f}=1730 \mathrm{~J} / \mathrm{kg} . \mathrm{K}, \rho_{f}=2200 \mathrm{~J} / \mathrm{kg} . \mathrm{K}, k_{f y}$ $=135 \mathrm{~W} / \mathrm{m} . \mathrm{K}$ (out-of-plane) and $k_{f z}=45 \mathrm{~W} / \mathrm{m} . \mathrm{K}$ (in-plane). The porosity for graphite foam is taken as $0.75^{1}$.

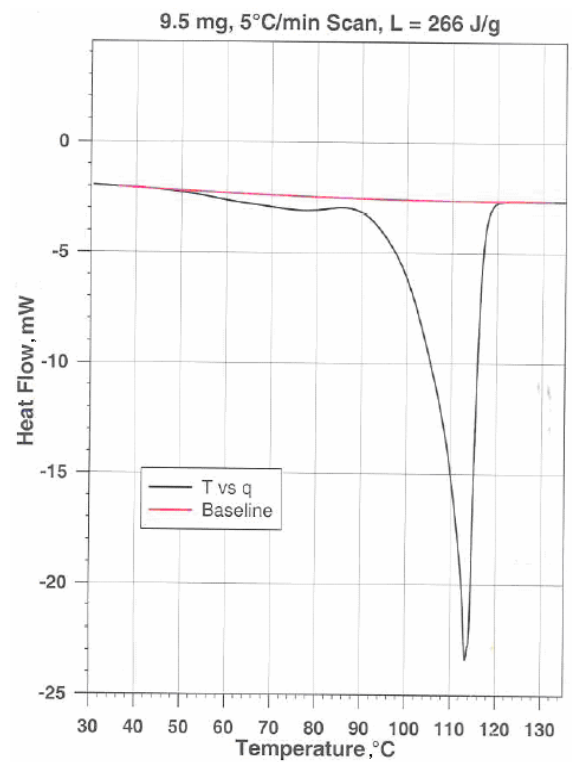

Figure 5. DSC curve of PCM

\footnotetext{
${ }^{1}$ http://www.poco.com/MaterialsandServices/ThermalMaterials/POCOFoam/PropertiesCharacteristics/tabid/130/Default.aspx
} 
The chosen PCM has a wide range of melting and so to use the latent heat of PCM effectively by simultaneously not sacrificing the narrow temperature operation of the device, an operating temperature range within the melting range of PCM is chosen where the latent heat is a maximum. This is achieved by integrating the DSC curve over a chosen operating range to obtain the area and hence the heat of fusion for that range of temperature. For example, if an operating range of $10 \mathrm{~K}$ is chosen, then there will be multiple options within the melting range ( $363 \mathrm{~K}-393 \mathrm{~K}$ ) but only one of them corresponds to a maximum heat of fusion. The best ranges of operation for all the possibilities within the melting range are found and an operating range of $8 \mathrm{~K}$ from $380 \mathrm{~K}$ to $388 \mathrm{~K}$ is chosen, where the latent heat, $h_{s f o}=138 \mathrm{~kJ} / \mathrm{kg}$.

Based on the above, $c_{p m}$ is redefined for this particular simulation as,

$$
c_{p m}=\frac{h_{s f o}}{\delta T_{o}}
$$

TC3, TC6 and TC7 in Fig. 6 shows the points of thermocouple tip locations in the actual columns that will be used for experiments. Two more arbitrary thermocouple locations TC1 and TC2 were chosen for the numerical simulation to monitor the outside wall temperature of the TES column. In Fig. 6, TC7 is located at $6 \mathrm{~mm}$ and TC2, TC3 and TC6 are located at $12 \mathrm{~mm}$, all in the vertical direction from the top surface of the foam (boundary 5 in Fig. 2). TC1 is chosen to be any point very close to the foam top surface. In the horizontal direction, TC1 and TC2 lie on the column wall outside, whereas TC3 lies on the boundary 2 in Fig. 2. TC6 lies at a distance of $W / 4$ and TC7 lies at a distance of $3 \mathrm{~W} / 8$ both from the column inside wall.

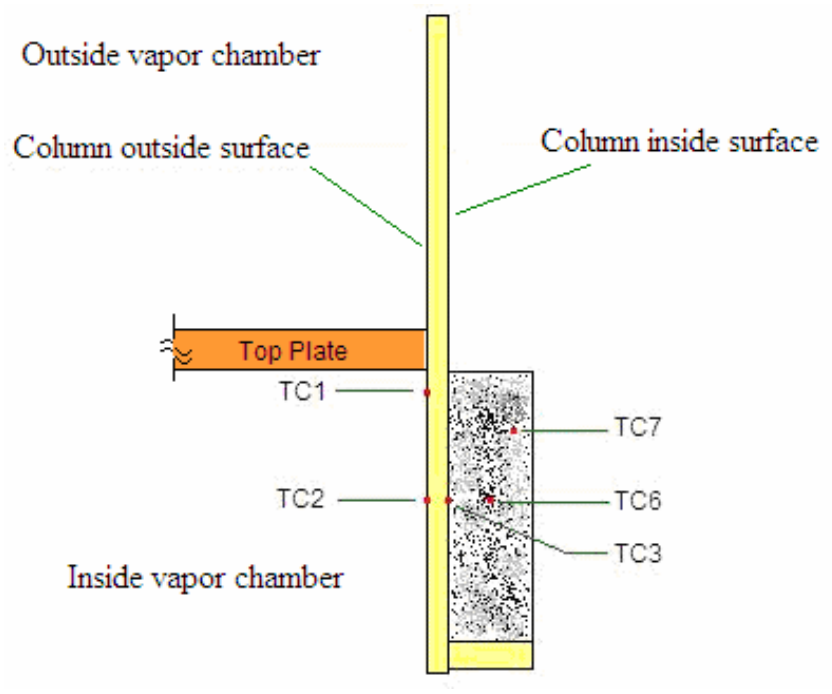

Figure 6. Thermocouple locations for the numerical model

A $T_{v}$ of $390 \mathrm{~K}, T_{0}$ of $380 \mathrm{~K}$ and a charging time of $15.5 \mathrm{~s}$ are chosen for all the simulations. Figure 7 shows the simulated time history of temperature at various thermocouple locations of Fig. 6.

The following observations and conclusions can be drawn from Fig. 7:

a) The wall temperature drops rapidly from an initial value of vapor temperature because of the presence of a heat sink (PCM) and then begins to rise slowly.

b) The dominant resistance during charging is from the condensate film. Once a steady state is reached (a long time after the end of charging), the film and PCM resistances become comparable ${ }^{1}$.

c) The presence of a varying thickness condensate film makes the PCM phase change problem twodimensional as expected.

d) The PCM temperature governs all other parameters like ' $T_{\text {wall }}$ ' and ' $\delta$ '.

e) Heat penetration into PCM is mostly from the top portion of the column wall where the film thickness is small because of a small thermal penetration resistance. This can be verified by comparing TC7 with TC6. 
f) The initial temperature choice ' $T_{0}$ ' of the PCM is appropriate since on an average, the PCM temperature rose from $380 \mathrm{~K}\left(107{ }^{\circ} \mathrm{C}\right)$ to $384.5 \mathrm{~K}\left(111.5^{\circ} \mathrm{C}\right)$, where the latent heat $h_{s f o}$ is a maximum within the PCM's entire melting range as discussed before.

g) PCM temperature at the end of charging time is still less than ' $T_{v}$ ' implies the vapor temperature will not rise before the end of charging period. This means that PCM is helpful in preventing the undue rise of temperature and pressure inside the vapor chamber.

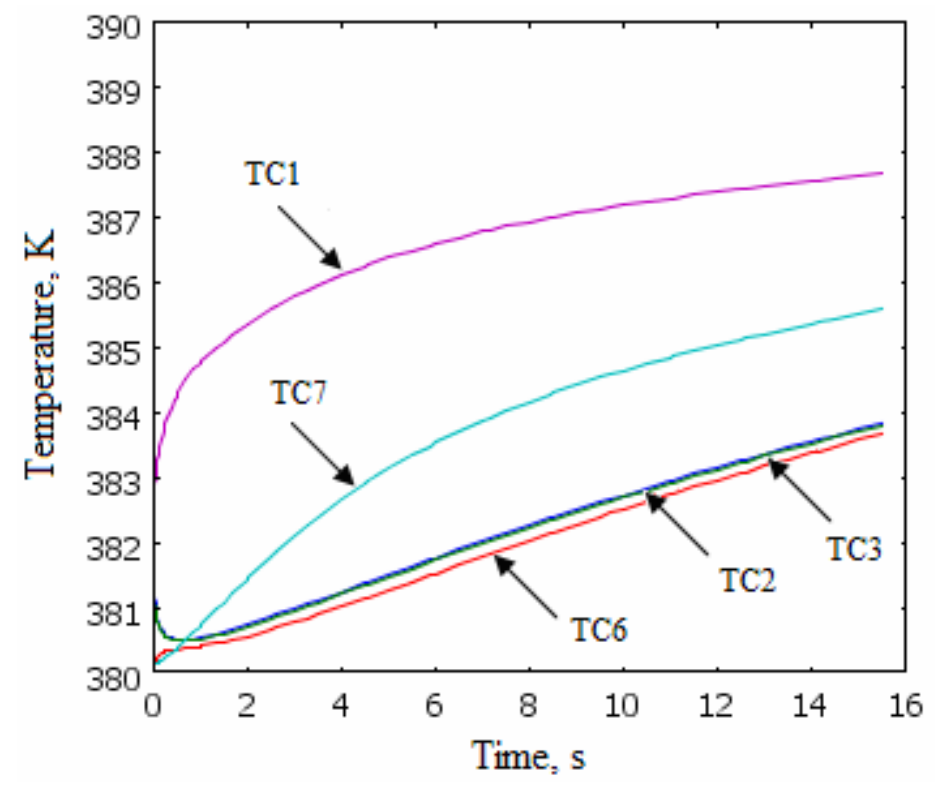

Figure 7. Temperature vs. time for PCM with a melting range

The temporal temperature distribution history for the current case under study can be seen in Fig. 8. Some of the conclusions of Fig. 7 can be clearly observed in Fig. 8. Figure 9 shows the time evolution of the condensate film during the charging time. The variable Time spans from 0 to $15.5 \mathrm{~s}$ and is the same in both the figures. In Fig. 9, it can be observed that the average film thickness at Time $=0$ is zero while at Time $=0.001 \mathrm{~s}$, it is about $81 \mu \mathrm{m}$ and at Time $=0.5 \mathrm{~s}, \delta$ on an average increases to $226 \mu \mathrm{m}$. This corresponds to the initial rapid cooling of the wall from ' $T_{v}$ ' at Time $=0$ to a much lower value at Time $=0.5 \mathrm{~s}$ as can be seen in Fig. 7. As $T_{\text {wall }}$ starts to rise again, the film thickness starts going down until the end of charging time to an average value of $190 \mu \mathrm{m}$.

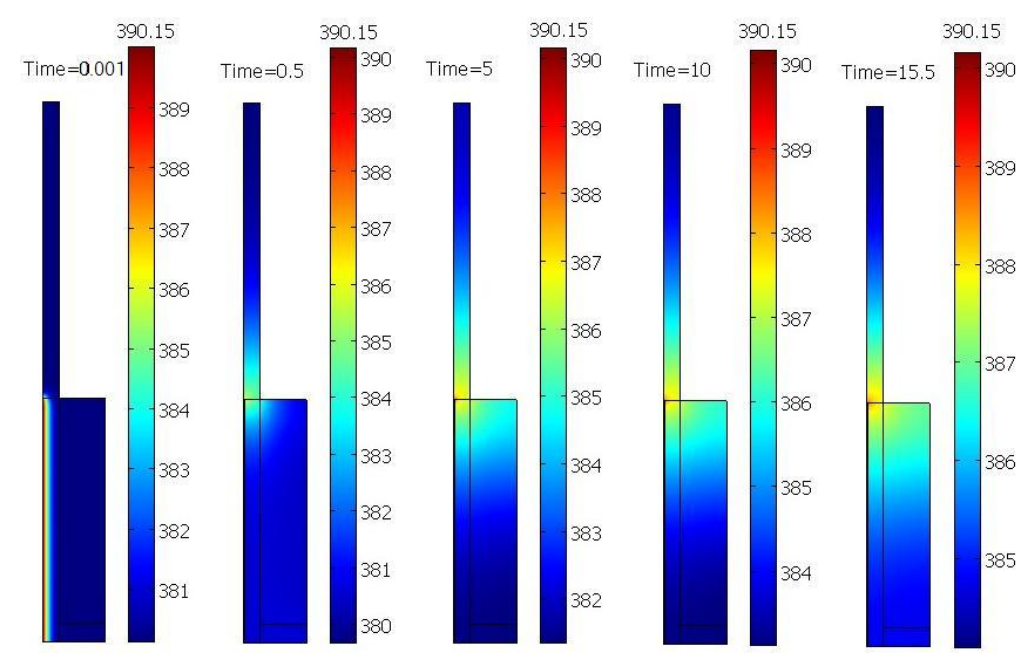

Figure 8. Temperature distribution in TES at various Time during charging 


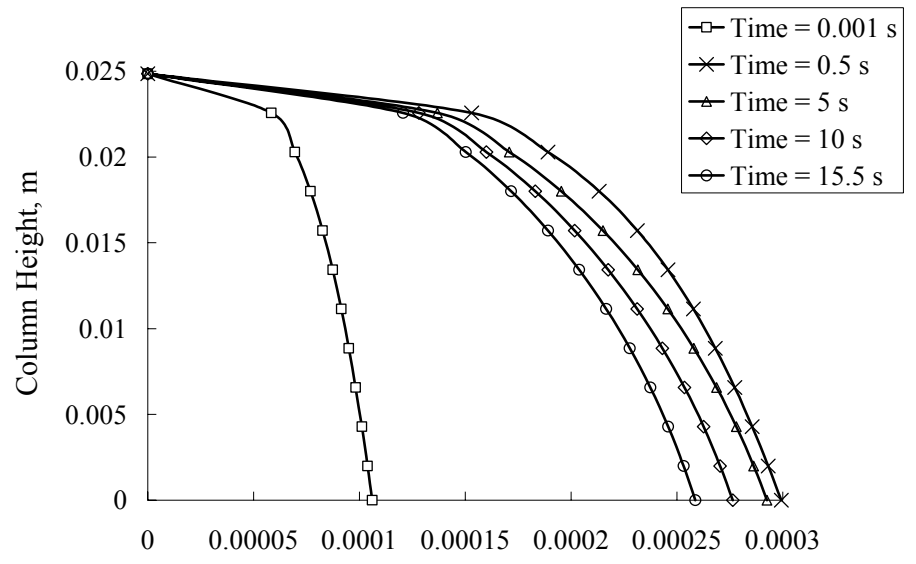

Film Thickness, m

Figure 9. Transient film profile history (film thickness at Time $=0$ is zero)

Using Fig. 7, the total power absorbed by all TES units is calculated as $235 \mathrm{~W}$ ( $3.63 \mathrm{~kJ}$ in $15.5 \mathrm{~s}$ ). The TES units in the experiment are designed to absorb $250 \mathrm{~W}$ for 15.5 seconds. Therefore, the numerical analysis validated the experimental design. It is also found that $81 \%$ of the total absorbed heat is by the PCM and foam composite, while the remaining $19 \%$ is by the encapsulating column walls. This shows the importance of including the column wall inertia effects in the simulation of the conjugate problem. In addition, inclusion of column walls in the model facilitates for simulating simultaneous heat removal on the condenser side (extended portion of the columns i.e., boundaries 6 and 7 in Fig. 2 that protrude outside of the vapor chamber as shown in Fig. 6).

\section{A. Importance of having TES in the VCTES heat sink}

The effect of presence of PCM on charging of the VCTES heat sink can be observed in Fig. 10, where the PCM was replaced with air for comparison with Fig. 7. It can be seen that the wall temperature drops down initially. However, because the sink in this case is not as effective as in Fig. 7, the wall temperature starts to rise rapidly and catches up with the vapor temperature even before the end of charging time.

If the vapor temperature was not fixed as in the current model, this will cause an increase in the vapor temperature and hence the pressure inside the chamber. It can also be observed by comparing Figs. 7 and 10 that in Fig. 10, the temperature rise is purely characteristic of transient heat conduction as in a solid but in Fig. 7, the temperature rise is in a more linear fashion because of PCM phase change.

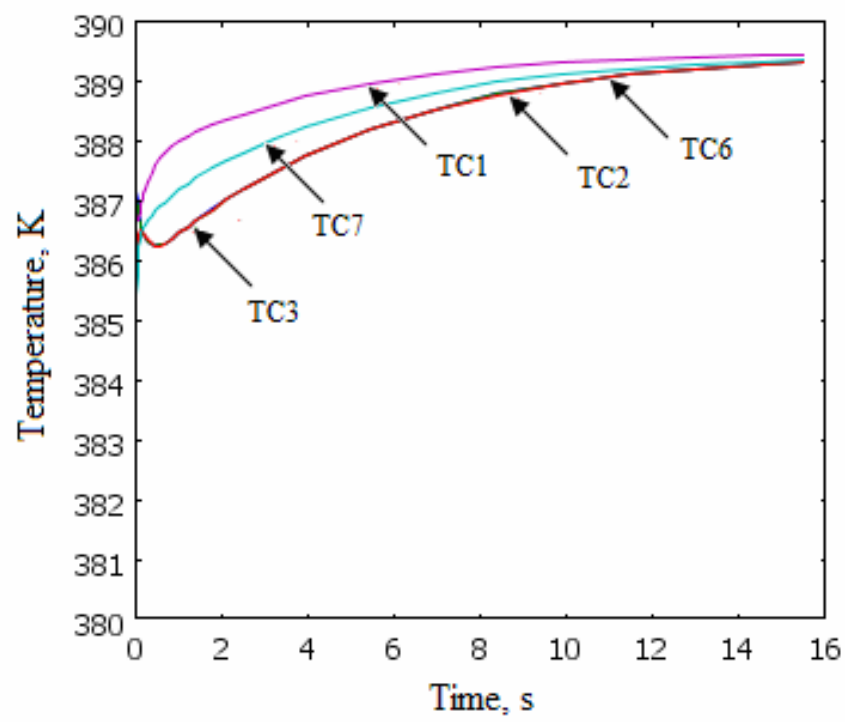

Figure 10. Temperature vs. time for TES columns with air (without PCM)

American Institute of Aeronautics and Astronautics 092407 


\section{B. Importance of pure PCM}

In Fig. 11, the effect of using a pure PCM is analyzed and plotted. Pure PCMs are characterized by isothermal melting (here, $386 \mathrm{~K}$ ) and hence the effect of full latent heat can be felt on the heat sink system. PCMs like the one used to simulate the case of Fig. 7, will have a reduction in the latent heat peak owing to its distribution over a melting range (shown in Fig. 5). The effect of melting range on the TES performance was studied and mentioned in Ref. 1. Heat capacity methods exhibit problems in simulating ideal isothermal melting and so usually enthalpy methods are used. Therefore, a $\delta T$ of $0.001 \mathrm{~K}$ is used to approximately model the phase change process for this case and the entire $h_{s f}$ of $266 \mathrm{~kJ} / \mathrm{kg}$ is assumed to be distributed over this $\delta T$. The temperature-time history of PCM in Fig. 11 is typical of pure PCMs. An initial cooling down of the wall temperature can be observed because of the presence of an effective heat sink (just like in Fig. 7). The wall temperature begins to rise again but remains almost a constant when the PCM reaches its melting point and continues to stay there even until the end of charging time.

The same initial temperature as chosen in the simulation of Fig. 7 was used even in the simulation of Fig. 11 and so we could observe rapid initial temperature rise in the beginning (the period of no PCM phase change). The advantage of pure PCMs can be very clearly seen if an initial temperature is chosen such that it is slightly less than the PCM melting temperature. For example, in the case of Fig. 11, if an initial temperature of $385.9 \mathrm{~K}$ were chosen, the entire operating temperature of PCM during charging period would have remained at $386 \mathrm{~K}$ and the wall temperature would have remained almost the same between $386.5 \mathrm{~K}$ and $387.5 \mathrm{~K}$. In Fig. 7, since the PCM has a melting range, it is inevitable to pick an initial temperature much less than the melting point peak to allow for the PCM temperature rise during melting.

Thus, it is evident that pure PCMs provide for better performance and near isothermal operation of the VCTES heat sink. It is interesting to note that the developed numerical model has little convergence problems even in solving an isothermal phase change problem, which is highly nonlinear compared to phase change problems with a melting range.

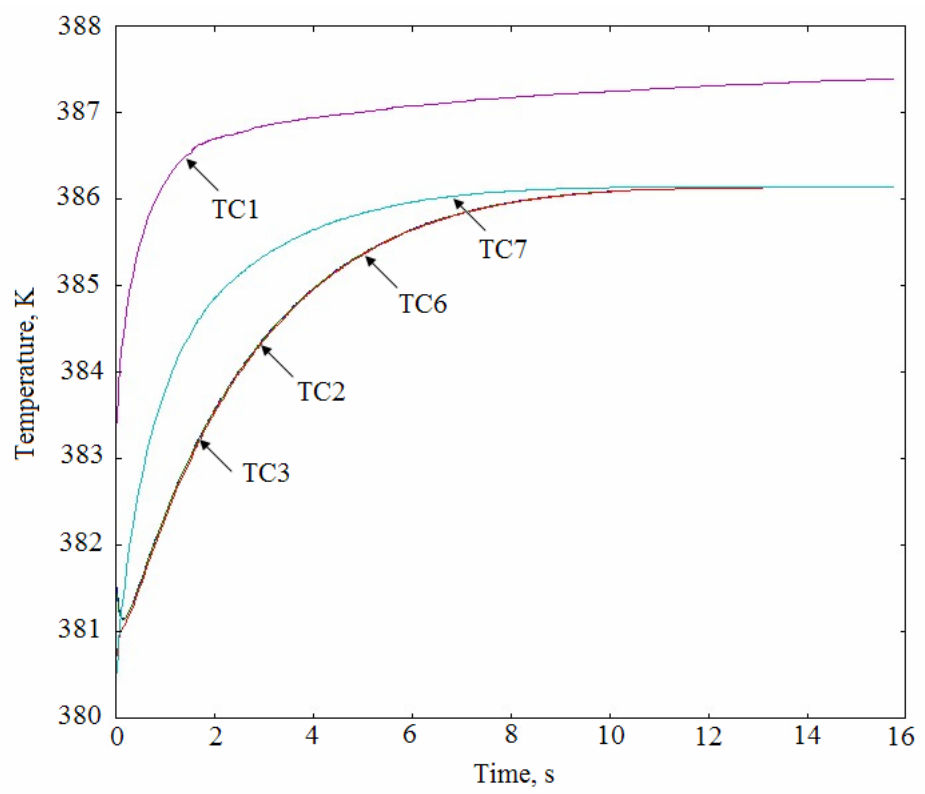

Figure 11. Temperature vs. time for pure PCM (with near-isothermal melting point)

\section{Importance of graphite foam in TES}

The developed numerical model was also used to study the importance of using foam in TES units. Accordingly, only PCM was assumed present in the columns without any thermal conduction-enhancing medium (graphite foam). The results are shown in Fig. 12. The wall temperature drops initially because of the presence of a sink in the form of copper walls and PCM but this drop is not quite as much as in Fig. 7. This is because in Fig. 12, the majority of the heat sink comprises only of copper walls because of the poor penetration of heat in the PCM sans graphite foam. This could be clearly observed in Fig. 12, where TC6 and TC7 are still at the initial temperature at the end of charging time indicating that no melting of PCM has taken place at those locations. Therefore, owing to its poor 
thermal conductivity, the resistance to heat transfer in PCM without graphite foam dominates the film condensation resistance during the transient charging process.

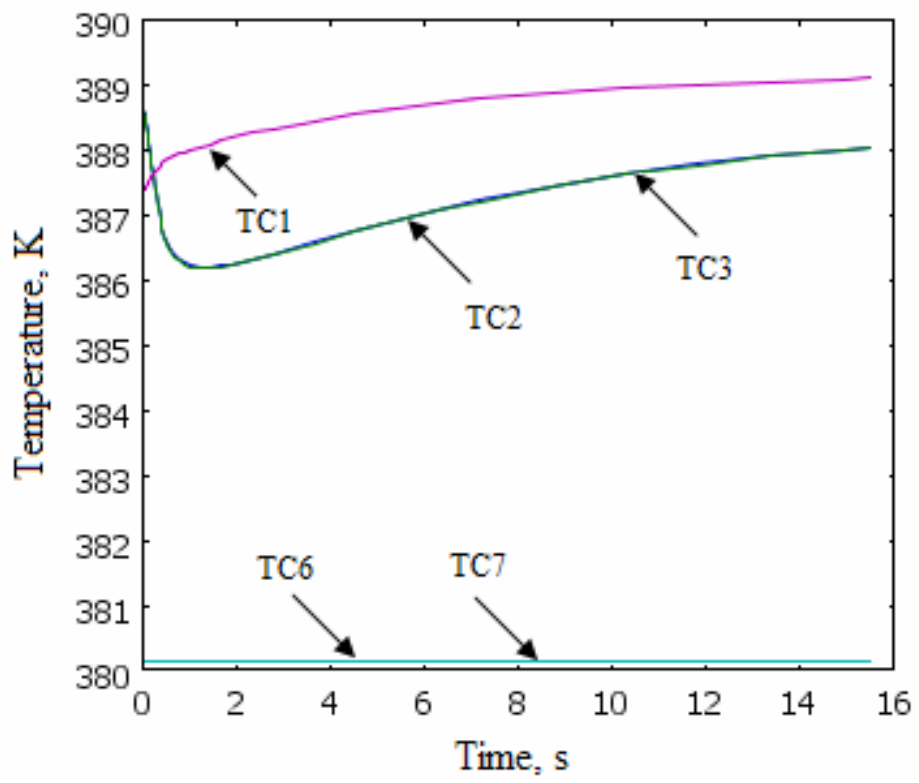

Figure 12. Temperature vs. time for TES columns without graphite foam

\section{Conclusion}

A concept of a dual latent heat sink intended for low thermal duty cycle electronic heat sink applications is presented in this paper. The key process of heat storage during the pulse heat generation period of the duty cycle for this heat sink comprises of transient simultaneous laminar film condensation of vapor and melting of an encapsulated phase change material in graphite foam. The problem is numerically analyzed including the wall inertia effects. An effective heat capacity formulation is employed for modeling the phase change problem and is solved using finite element method.

The results of the developed model showed that the concept is effective in preventing undue temperature rise of the heat source. The advantage of having a dual latent phase change phenomenon is shown as compared to single latent heat sink by assuming the lack of PCM in the design. It is also numerically shown that pure PCM exhibits a better performance. The significance of having a thermal conductivity enhancing medium for PCM is also shown. As expected, graphite foam plays a very crucial role in transferring heat to the PCM and thus helps in rapid charging.

It is found that during the transient operation of a VCTES heat sink, the key impediment to rapid heat absorption comes from the condensate film. Because of the condensate profile, the PCM phase change problem becomes twodimensional and the temperature gradient in the interfacial solid wall in the height direction is large. It is also observed that for PCMs with a melting range, selection of a right operating range and initial temperature are crucial.

\section{Acknowledgments}

The authors sincerely wish to thank Michele Puterbaugh, the program manager at Universal Technology Corporation, Dayton, $\mathrm{OH}$ for funding the work.

\section{References}

${ }^{1}$ Kota, K., Chow, L., Du, J., Kapat, J., Leland, Q. and Harris, R., "Conceptual Design of a Dual Latent Heat Sink for Thermal Management of Pulse Heat Generating Electronic Systems," Proceedings of $39^{\text {th }}$ AIAA Thermophysics Conference, AIAA, Reston, VA, 2007.

${ }^{2}$ Contreras, W., and Thorsen, R. S., "Transient Melting of a Solid Heated by a Condensing Saturated Vapor - Case 1: Negligible Interface Curvature,” Journal of Heat Transfer, Vol. 97, 1975, pp. 570-575.

${ }^{3}$ Galamba, D., "Some Aspects of Simultaneous Melting-Condensation on Vertical Surfaces," Ph. D. Dissertation, Department of Mechanical and Aerospace Engineering, University of California, Los Angeles, CA, 1985.

14

American Institute of Aeronautics and Astronautics 092407 
${ }^{4}$ Galamba, D. and Dhir, V. K., "Transient Simultaneous Condensation and Melting of a Vertical Surface," ASME Journal of Heat Transfer, Vol. 107, No. 4, 1985, pp. 812-818.

${ }^{5}$ Galamba, D. and Dhir, V. K., "Transient Condensation - Melting of a Subcooled Vertical Surface," Numerical Heat Transfer, Vol. 15, 1988, pp. 33-65.

${ }^{6} \mathrm{Du}, \mathrm{J} .$, Chow, L., and Leland, Q., "Optimization of High Heat Flux Thermal Energy Storage with Phase Change Materials," Proceedings of 2005 ASME International Mechanical Engineering Congress and Exposition, Orlando, FL, 5-11 November 2005.

${ }^{7}$ Klett, J., "High Thermal Conductivity Graphite Foam," URL: www.ms.ornl.gov/researchgroups/cmt/foam/foams.htm.

${ }^{8}$ Chen, H., and Chang, S., "Coupling Between Laminar Film Condensation and Natural Convection on Opposite Sides of a Vertical Plate," International Journal for Numerical Methods in Fluids, Vol. 24, 1997, pp. 319-336.

${ }^{9}$ Char, M., and Lin, J., "Conjugate Film Condensation and Natural Convection Between Two Porous Media Separated by a Vertical Plate," Acta Mechanica, Vol. 148, 2001, pp. 1-15.

${ }^{10}$ Krishnan, S., Murthy, J., Garimella, S., "A Two-Temperature Model for the Analysis of Passive Thermal Control Systems," Journal of Heat Transfer, Vol. 126, 2004, pp. 628-637.

${ }^{11}$ Lee, D., and Vafai, K., "Analytical Characterization and Conceptual Assessment of Solid and Fluid Temperature Differentials in Porous Media," International Journal of Heat and Mass Transfer, Vol. 42, 1999, pp. 423-435.

12،"What is Pocofoam?," URL: http://www.poco.com/us/Thermal/foam.asp.

${ }^{13}$ Incropera, F. P., DeWitt, D. P., Bergman, T. L., Lavine, A. S., Fundamentals of Heat and Mass Transfer, $6^{\text {th }}$ ed., John Wiley \& Sons, Inc., NJ, 2007.

${ }^{14}$ Morgan, K., "A Numerical Analysis of Freezing and Melting With Convection," Computer Methods in Applied Mechanics and Engineering, Vol. 28, No. 3, pp. 275-284.

${ }^{15}$ Hsiao, J. S. and Chung, B. T. F., "An Efficient Algorithm for Finite Element Solution to Two-Dimensional Heat Transfer With Melting and Freezing," Proceedings of $22^{\text {nd }}$ ASME National Heat Transfer Conference, ASME, New York, NY, 1984.

${ }^{16}$ Cao, Y. and Faghri, A., "A Numerical Analysis of Phase-Change Problems Including Natural Convection," ASME Journal of Heat Transfer, Vol. 112, No. 3, 1990, pp. 812-816.

${ }^{17}$ Voller, V. R. and Cross, M., "Accurate Solutions of Moving Boundary Problems Using the Enthalpy Method," International Journal of Heat and Mass Transfer, Vol. 24, 1981, pp. 545-556.

${ }^{18}$ Voller, V. R., "Implicit Finite Difference Solutions of the Enthalpy Formulation of Stefan Problems," IMA Journal of Numerical Analysis, Vol. 5, 1985, pp. 201-214.

${ }^{19}$ Voller, V. R., Cross, M. and Markatos, N. C., "An Enthalpy Method for Convection/Diffusion Phase Change," International Journal for Numerical Methods in Engineering, Vol. 24, 1987, pp. 271-284.

${ }^{20}$ Voller, V. R., "Fast Implicit Finite Difference Method for the Analysis of Phase Change Problems," Numerical Heat Transfer B, Vol. 17, 1990, pp. 155-169.

${ }^{21}$ Paradela, F., Queimada, A., Marrucho, I., Neto, C., and Coutinho, J., "Modeling the Thermal Conductivity of Pure and Mixed Heavy n-Alkanes Suitable for the Design of Phase Change Materials," International Journal of Thermophysics, Vol. 26, No. 5, 2005, pp. 1461-1475.

${ }^{22}$ Bart, G. C. J. and van der Laag, P. C., "Modeling of Arbitrary-shaped Specific and Latent Heat Curves in Phase Change Storage Simulation Routines," ASME Journal of Solar Energy Engineering, Vol. 112, 1990, pp. $29-33$.

${ }^{23}$ Sparrow, E. M. and Siegel, R., "Transient Film Condensation”, Journal of Applied Mechanics, Vol. 26, 1959, pp. $120-121$.

${ }^{24}$ Reed, J. G., Gerner, F. M., Tien, C. L., "Transient Laminar-Film Condensation on a Vertical Plate", Journal of Thermophysics and Heat Transfer, Vol. 2, No. 3, 1988, pp. 257-263.

${ }^{25}$ Chung, P. M., "Unsteady Laminar Film Condensation on Vertical Plate”, ASME Journal of Heat Transfer, 1963, pp. 63-70.

${ }^{26}$ COMSOL Multiphysics, Modeling Package, Ver. 3.3, COMSOL, Inc., Burlington, MA, 1997-2007.

${ }^{27}$ MATLAB - The Language of Technical Computing, The Mathworks, Inc., Natick, MA, 1994-2007.

${ }^{28}$ Mesalhy, O., Lafdi, K., Elgafy, A., "Carbon Foam Matrices Saturated with PCM for Thermal Protection Purposes," Carbon, Vol. 44, 2006, pp. 2080-2088. 